\title{
INFARTO AGUDO DO MIOCÁRDIO COM SUPRADESNÍVEL DO SEGMENTO ST: HÁ ESPAÇO PARA O USO DE FIBRINOLÍTICO?
}

\section{ACUTE MYOCARDIAL INFARCTION WITH ST ELEVATION: IS THERE SPACE FOR THE USE OF FIBRINOLYTIC THERAPY?}

\author{
Bruno Ribeiro $^{1}$; Bianca Eliza Hoekstra ${ }^{2}$; Elise Souza dos Santos Reis ${ }^{3}$; Mario Augusto \\ Cray da Costa ${ }^{4}$ \\ ${ }^{1}$ Graduando do curso de Medicina da Universidade Estadual de Ponta Grossa. \\ E-mail: bruno_rb@msn.com \\ ${ }^{2}$ Graduanda do curso de Medicina da Universidade Estadual de Ponta Grossa. \\ E-mail: bieliza@hotmail.com \\ ${ }^{3}$ Médica cardiologista e hemodinamicista da Santa Casa de Misericórdia de Ponta Grossa/ \\ Paraná e professora da disciplina de Clínica Médica I e II da Universidade Estadual de \\ Ponta Grossa. E-mail: essreis@brturbo.com.br \\ ${ }^{4}$ Médico cirurgião cardiovascular da Santa Casa de Misericórdia de Ponta Grossa/Paraná e \\ professor da disciplina de Clínica Cirúrgica da Universidade Estadual de Ponta Grossa. \\ E-mail: drmarioaugusto@uol.com.br
}

Data de recebimento: $18 / 03 / 2014$

Data da aprovação: 01/06/2014

\section{RESUMO}

A maioria das mortes por infarto agudo do miocárdio ocorre nas primeiras horas de manifestação da doença. O objetivo deste estudo consiste na reafirmação de que quando o atraso da chegada do paciente a um serviço de hemodinâmica é evidente, a utilização da terapêutica fibrinolítica precoce é benéfica.

O estudo analítico observacional do tipo caso-controle realizou-se por meio da análise de 751 prontuários do Serviço de Hemodinâmica da Santa Casa de Misericórdia de Ponta Grossa/Paraná entre 2007 e 2012. Para comparação das diferenças na sobrevida e mortalidade, os pacientes foram distribuídos em grupos de acordo com a terapia primária de reperfusão adotada (fibrinolítico ou angioplastia), o tempo de início do tratamento e a classificação clínica de Killip-Kimbal durante a internação.

Dentre outros resultados, confirmamos que se a perspectiva do paciente ser submetido à angioplastia precoce for desfavorável, autilização do trombolítico é indicada ( $\mathrm{p}=0.008)$ com oddsratio de 12, mostrando que a chance do paciente desenvolver Killip II, III ou IV é 12 vezes maior na angioplastia após 12 horas quando comparada ao trombolítico em menos de 6 horas de evolução do IAM.

Conseguimos estabelecer a importância da utilização da terapia fibrinolítica sempre que o retardo para a realização da angioplastia for elevado e que com esta conduta se garante melhor evolução clínica do paciente.

Palavras-chave:Infarto do Miocárdio.Angioplastia.Fibrinólise.

\begin{abstract}
Most deaths from acute myocardial infarction occur in the early hours of the disease onset. The objective of this study is the reaffirmation that the use of earlier fibrinolytic therapy is beneficial when the delayed arrival of the patient to a hemodynamic service is clear.
\end{abstract}


An analytical observational case-control study was conducted by analyzing 751 medical records of the Hemodynamics Service of Santa Casa de Misericordia de Ponta Grossa / Paraná in 2007 to 2012. To compare the differences in survival and mortality, patients were divided into groups according to the primary reperfusion therapy adopted (fibrinolysis or angioplasty), the time of initiation of the treatment and the clinical classification of Killip-Kimball during hospitalization.

Among other results, we confirmed that if the perspective of the patient to get an early angioplasty is unfavorable, it is better to use the fibrinolytic therapy $(p=0.008)$ with an odds ratio of 12. The chance of the patient developing Killip II, III or IV is 12 times higher in the angioplasty after 12 hours compared to fibrinolytic therapy in 6 hours or less of the onset of AMI.

We were able to establish the importance of the use of fibrinolytic therapy whenever there is a delay for performing angioplasty and that the use of it ensures a better clinical outcome.

Keywords: Myocardial Infarction. Angioplasty. Fibrinolysis.

\section{INTRODUÇÃO}

As doenças cardiovasculares (DCV), principalmente o infarto agudo do miocárdio (IAM) e o acidente vascular encefálico (AVE), atualmente representam a principal causa de mortalidade no mundo, e nos últimos anos estão associadas ao acometimento de pacientes cada vez mais jovens e com o aumento da incidência nos países em desenvolvimento (MURRAY, 1996; WHO, 2010; 2011).

A maioria das mortes por IAM ocorre nas primeiras horas de manifestação da doença.Em 40 a $65 \%$ dos casos o óbito acontece na primeira hora e, aproximadamente, em $80 \%$ dos casos, nas primeiras 24 horas, e grande parte fora do ambiente hospitalar, geralmente sem assistência médica (PIEGAS et al., 2009).

Os protocolos estabelecidos para o tratamento de um IAM com supradesnivelamento de ST (IAMCSST) geralmente são focados na diminuição do tempo de tratamento após a entrada no hospital. Contudo, este enfoque falha ao desconsiderar o período pré-hospitalar e o enorme número de mortes que ocorrem neste intervalo (Ibidem).

O objetivo principal do tratamento de pacientes que apresentam IAMCSST é a recanalização rápida e completa da artéria coronária ocluída para que se preserve o tecido muscular adjacente a ela e para manter a normalidade funcional do coração (DENKTAS et al., 2011).

Infelizmente, é frequente a chegada de pacientes com tempo elevado de evolução do IAM que aguardam pela intervenção coronária percutânea (ICP) primária e que poderiam ter realizado terapia fibrinolítica no local de origem sem acarretar danos evitáveis ao miocárdio e, consequentemente, à sobrevida do paciente.
Esse estudo consiste na avaliaçãoda mortalidade e evolução clínica entre os grupos: reperfusão química (fibrinolítico) e mecânica (angioplastia primária) de acordo com o momento da intervenção terapêutica e intenta deixar claro por meio dos resultados que na impossibilidade de um paciente ser transferido emmenos de duas horas para um serviço com hemodinâmica 24 horas, o uso de fibrinolítico deve ser instituído.

\section{Materiaise métodos}

A pesquisa de caráter analítico observacional do tipo caso-controle contou com a análise de dados coletados de 751prontuários do Serviço de Hemodinâmica da Santa Casa de Misericórdia de Ponta Grossa/Paraná (SCMPG/PR) que apresentavam como diagnóstico de entrada infarto agudo do miocárdio ou infarto agudo do miocárdio recorrente entre2007 e 2012.

Foram incluídos na pesquisa os prontuários dos pacientes atendidos no pronto atendimento da SCMPG/PR com diagnóstico de infarto agudo do miocárdio com supradesnivelamento do segmento ST maior que $2 \mathrm{~mm}$ nas derivações do plano horizontal e maior que $1 \mathrm{~mm}$ nas derivações do plano frontal.

Foram excluídos os prontuários preenchidos de maneira incompleta, que inviabilizavam a distribuição destes pacientes nos grupos propostos, e aqueles que realizavam procedimentos eletivos e/ou que em concomitância com o IAM possuíam: bloqueio de ramo esquerdo de tempo desconhecido, miocardiopatia, dissecção aguda de aorta ou pericardite.

Os pacientes foram separados em dois grandes grupos: Reperfusão Química(RQ)e Reperfusão Mecânica (RM), de acordo com a conduta inicial realizada. 
Ambos os grupos foram subdivididos de acordo com o tempo entre o início da dor e o momento da intervenção em subgrupos: menor que 6 horas, de 6 a 12 horas e maior que 12 horas (Quadro 1).
3) Evolução hospitalar:

a) Incidência de MACE (Morte,IAM, AVE e necessidade de revascularização);

b) Necessidade de angioplastia de resgate;

Quadro 1 -Distribuição da amostra pelo tempo de início da intervenção

\begin{tabular}{|c|c|c|c|c|c|c|}
\hline & \multicolumn{3}{|c|}{ Reperfusão Química (Fibrinolítico) } & \multicolumn{3}{c|}{ Reperfusão Mecânica (Angioplastia) } \\
\hline Tempo & $<6$ horas & $6-12$ horas & $>12$ horas & $<6$ horas & $6-12$ horas & $>12$ horas \\
\hline
\end{tabular}

Os seguintes dados dos pacientes foram obtidos por meioda observação de prontuários (prontuário eletrônico, Tasy):

1) Características clínicas: Sexo, idade, antecedentes pessoais (hipertensão arterial sistêmica, diabetes mellitus, dislipidemia, tabagismo, histórico familiar e IAM ou revascularização do miocárdio prévios), artéria coronária acometida, tempo de internamento e evolução do paciente (alta ou óbito);

2) Dados específicos de cada procedimento:

a) Angioplastia: sucesso angiográfico definido pela abertura da artéria e fluxo distal (escala TIMI II ou III);

b) Fibrinolítico: diminuição da intensidade da dor, redução do supradesnivelamento ST, arritmia de reperfusão. c) Classificação clínica de Killip-Kimball(KK) durante a internação:

- Classe I - ausência de sinais de insuficiência cardíaca;

- Classe II - presença de estertores crepitantes nos pulmões e pressão venosa central elevada;

- Classe III - franco edema agudo de pulmão;

- Classe IV - choque cardiogênico ou hipotensão (medida como pressão arterial sistólica $<90$ $\mathrm{mmHg}$ ) e evidência de vasoconstricção periférica (oligúria, cianose e/ou sudorese).

Após a coleta dos dados e separação dos pacientes entre os grupos de RQ e RM, comparou-se os valores obtidos nos dois grupos para que ficasse clara a similaridade da amostra. (Tabela 1).

Tabela 1 - Características de base dos pacientes avaliados

\begin{tabular}{|c|c|c|c|}
\hline & $\begin{array}{c}\text { Reperfusão Química } \\
\text { (Fibrinolítico) }\end{array}$ & $\begin{array}{l}\text { Reperfusão Mecânica } \\
\text { (Angioplastia) }\end{array}$ & \\
\hline & $\mathrm{n}=18$ & $\mathrm{n}=\mathbf{8 0}$ & $p$ \\
\hline \multicolumn{4}{|l|}{ Idade } \\
\hline Média (DP) & $58.94(12,55)$ & $57,58(13,10)$ & 0,68 \\
\hline \multicolumn{4}{|l|}{ Sexo } \\
\hline Maculino & $13(72.2 \%)$ & $63(78,7 \%)$ & \\
\hline Feminino & $5(28,8 \%)$ & $17(21,3 \%)$ & 0,54 \\
\hline \multicolumn{4}{|l|}{ Fatores de risco } \\
\hline HAS & $14(77,7 \%)$ & $48(60 \%)$ & 0,15 \\
\hline DM2 & $5(27,7 \%)$ & $14(17 \%)$ & 0,31 \\
\hline Dislipidemia & $9(50 \%)$ & $20(25 \%)$ & 0,03 \\
\hline Tabagismo & $8(44,4 \%)$ & $30(37,5 \%)$ & 0,58 \\
\hline História familiar & $6(33,3 \%)$ & $14(17,5 \%)$ & 0,13 \\
\hline \multicolumn{4}{|l|}{ Antecedentes } \\
\hline IAM prévio & $4(22,2 \%)$ & $8(10 \%)$ & 0,15 \\
\hline \multicolumn{4}{|l|}{ Artéria acometida } \\
\hline DA & $10(55,5 \%)$ & $41(51,2 \%)$ & 0,74 \\
\hline CD & $9(50 \%)$ & $29(36,2 \%)$ & 0,27 \\
\hline
\end{tabular}




\begin{tabular}{|c|c|c|c|}
\hline & $\begin{array}{l}\text { Reperfusão Química } \\
\text { (Fibrinolítico) }\end{array}$ & $\begin{array}{l}\text { Reperfusão Mecânica } \\
\text { (Angioplastia) }\end{array}$ & \\
\hline $\mathbf{C X}$ & $2(11,1 \%)$ & $9(11,2 \%)$ & 1 \\
\hline & & & \\
\hline $\begin{array}{l}\text { Presença de critérios } \\
\text { de reperfusão }\end{array}$ & $16(88,8 \%)$ & - & $\mathrm{n} / \mathrm{a}$ \\
\hline Angioplastia de resgate & $6(33,3 \%)$ & - & $\mathrm{n} / \mathrm{a}$ \\
\hline \multicolumn{4}{|l|}{ Timi Flow } \\
\hline $\mathbf{0}$ & - & $5(6,2 \%)$ & $\mathrm{n} / \mathrm{a}$ \\
\hline 1 & - & $1(1,2 \%)$ & $\mathrm{n} / \mathrm{a}$ \\
\hline 2 & - & $6(7,5 \%)$ & $\mathrm{n} / \mathrm{a}$ \\
\hline 3 & - & $68(85 \%)$ & $\mathrm{n} / \mathrm{a}$ \\
\hline \multicolumn{4}{|l|}{ Tempo transcorrido } \\
\hline$<6$ horas & $13(75 \%)$ & $39(49 \%)$ & 0,07 \\
\hline Entre 6 e 12 horas & $5(25 \%)$ & $13(17 \%)$ & 0,25 \\
\hline$>12$ horas & $0(\%)$ & $28(34 \%)$ & 0,002 \\
\hline \multicolumn{4}{|l|}{ Tempo de internação } \\
\hline Média (DP) & $7,89(4,8)$ & $7,03(4,6)$ & 0,47 \\
\hline \multicolumn{4}{|l|}{ Killip(fase hospitalar) } \\
\hline I & $14(77,7 \%)$ & $57(71,2 \%)$ & 0,57 \\
\hline II & $4(22 \%)$ & $15(18,7 \%)$ & 0,73 \\
\hline III & $0(0 \%)$ & $4(5 \%)$ & 0,33 \\
\hline IV & $0(0 \%)$ & $4(5 \%)$ & 0,33 \\
\hline \multicolumn{4}{|l|}{ Evolução } \\
\hline Alta & $18(100 \%)$ & $71(88,7 \%)$ & 0,13 \\
\hline Óbito & $0(0 \%)$ & $9(11,3 \%)$ & 0,13 \\
\hline
\end{tabular}

Após distribuir os pacientes em subgrupos indicados no Quadro 1, buscou-se identificar as diferenças existentes na mortalidade e evolução clínica de acordo com os tipos e os diferentes tempos de início das intervenções terapêuticas.

O estudo foi aprovado pelo Comitê de Ética e Pesquisa em Seres Humanos da Universidade Estadual de Ponta Grossa (UEPG), no Paraná, sob o parecer n ${ }^{\circ}$ 159.535, submetido em 27 de novembro de 2012.

O Termo de Consentimento Livre e Esclarecido não foi necessário devido à natureza analítica observacional do estudo.

\section{Análise estatística}

Todos os dados foram testados quanto ao padrão de distribuição (paramétrico ou não). As diferenças estatísticas das médias entre os grupos foram determinadas utilizando-se os testes $t$ de Student bicaudal para distribuições paramétricas e as variáveis contínuas são apresentadas em médias \pm desvio padrão. Para a análise de diferenças entre valores encontrados foi utilizado o teste do quiquadrado com correção de Yates. Para a análise estatística e a realização dos cálculos utilizou-se o software GraphPad. As diferenças foram consideradas significativas quando o $p$ obtido foi menor do que 0.05 .

\section{Resultados}

A amostra foi composta por 98 pacientes, sendo 18 do grupo da Reperfusão Química e 80 do grupo da Reperfusão Mecânica.

$\mathrm{Na}$ Tabela 1 são discriminadas todas as características de base encontradas nos prontuários dos pacientes divididos entre estes grupos antes de 
subdividi-los em grupos menores de acordo com o tempo do início da intervenção.

As médias de idade e a distribuição entre os sexos foram similares, não havendo diferença estatisticamente significativa entre os grupos. Para o grupo do fibrinolítico, a média de idade foi de 58,94 $\pm 12,55$ anos, e no grupo de angioplastia, $(p=0,68)$ de 57,58 $\pm 13,10$ anos. Em ambos os grupos $(p=0.54)$, houve uma clara predominância do sexo masculino $(72,2 \%$ na RQ e $78,7 \%$ na RM).

Entre os fatores de risco avaliados, nota-se nos dois grupos $(p=0,15)$ a hipertensão arterial sistêmica (HAS) como a mais prevalente, presente em 14 pacientes $(77 \%)$ da RQ e em 48 pacientes $(60 \%)$ da RM, ou seja, em $63 \%$ de toda a amostra.

A média de tempo de internação para os dois grupos também não apresentou diferença estatisticamente significativa $(p=0,47)$, visto que para o grupo da RQ obteve-se uma média de 7,89 $\pm 4,8$ dias e para o grupo da RM uma média de 7,03 \pm 4,6 dias.

Diferenças estatisticamente significativas foram encontradas nos dados da Tabela 1 em apenas duas situações:

1) A presença de dislipidemia, um dos fatores de risco para IAM analisado, teve diferença estatisticamente significativa entre os dois grupos. No grupo do fibrinolítico, estava presente em 9 dos 18 pacientes $(50 \%)$ e, no grupo da angioplastia, em 20 dos 80 pacientes $(25 \%, p=0,03)$. Esta diferença estatística, entretanto, pode ser explicada pela descrição insatisfatória dos pacientes nos prontuários analisados, uma vez que é de responsabilidade médica a inserção dos dados de forma correta e integral;
2)A diferença entre o número de pacientes nos subgrupos com mais de 12 horas de evolução do IAM também foi estatisticamente significativa, já que para nenhum paciente foi indicada a terapia fibrinolítica, e 28 pacientes foram submetidos à angioplastia primária após 12 horas $(0 \%$ x 34\%, $p=0.002)$. Aqui devemos levar em conta que na IV Diretriz da Sociedade Brasileira de Cardiologia sobre Tratamento do Infarto Agudo do Miocárdio com Supradesnível do Segmento ST, é demonstrado que não há vantagem na utilização da terapia fibrinolítica após 12 horas, não sendo prescrita após este intervalo de tempo,a menos que o paciente ainda apresente precordialgia.

Algumas características abordadas na Tabela 1 não puderam ser comparadas por se adequarem a apenas um dos grupos.

Para determinar as diferenças na mortalidade e evolução clínica dos pacientes de acordo com os tipos e diferentes tempos de início das intervenções terapêuticas, e ainda, para comprovar a prescrição da terapia fibrinolítica precoce em casos de atraso eminente do encaminhamento do paciente até o serviço de hemodinâmica para realização da angioplastia primária, comparou-se: para a evolução clínica, a classificação evolutiva de Killip-Kimball dos pacientes que obtiveram como terapia de reperfusão primária o fibrinolítico em menos de 6 horas $(n=13)$ com aqueles que se submeteram a ICP primária após 12 horas de evolução da doença $(n=28)$, conforme a Tabela 2, e foi obtido um $p=0.07$; e para a mortalidade, utilizando-se os mesmos grupos, os desfechos dos pacientes entre óbito ou alta, como é mostrado na Tabela 3 , com $p=0.38$.

Tabela 2 -Relação do escore de Killip durante a internação com o procedimento realizado

\begin{tabular}{c|c|c|c|}
\hline & Trombolítico $<$ 6 horas & Angioplastia $>$ 12 horas & $\mathbf{n}=\mathbf{2 8}$ \\
\hline Killip & $\mathbf{n = 1 3}$ & & \\
\hline I & & $14(50 \%)$ & \\
\hline II & $12(92,3 \%)$ & $9(32,1 \%)$ & \\
\hline III & $1(7,7 \%)$ & $2(7,2 \%)$ & 0,07 \\
\hline IV & $0(0 \%)$ & $3(10,7 \%)$ & \\
\hline
\end{tabular}


Tabela 3 - Avaliação da mortalidade de acordo com o procedimento realizado

\begin{tabular}{cccc}
\hline & Trombolítico $<\mathbf{6}$ horas & Angioplastia $>\mathbf{1 2}$ horas & $\boldsymbol{p}$ \\
\hline Evolução & $\mathbf{n = 1 3}$ & $\mathbf{n = 2 8}$ & \\
\hline Alta & & $24(85,7 \%)$ & 0,38 \\
\hline Óbito & $13(100 \%)$ & $4(14,3 \%)$ & 0 \\
\hline
\end{tabular}

Em uma análise post-hoc, dividiram-se os pacientes em Killip I (sem sinais de insuficiência cardíaca), e foram agrupados aqueles com classificação KK II, III e IV de Killip-Kimball (maior grau de gravidade). Ao ser refeita a comparação entre os que realizaram fibrinolítico em menos de 6 horas e os que se submeteram a angioplastia após 12 horas da doença (Tabela 4), percebe-se que apenas um paciente $(7,7 \%)$ do grupo do fibrinolítico em menos de 6 horas evoluiu para KK>I. Já no grupo da angioplastia após 12 horas, 14 pacientes $(50 \%)$ foram classificados como Killip entre II e IV, resultando em um odds ratio (OR) de 12 (IC 95\%: 1.36-105.13, $\mathrm{p}=0.008$ ). a terapêutica estabelecida pelos protocolos durante o período pré-hospitalar e dentro do tempo adequado (CALUZA et al., 2012).

Infelizmente, o preço a pagar nessas situações de falta de organização no atendimento geral é uma morbimortalidade significantemente mais elevada (Ibidem).

Vários estudos (ARMSTRONG et al., 2006; BORGIA et al., 2010; HUYNH et al., 2009; LAMBERT et al., 2010; VAN DE WERF et al., 2008) comprovam que a intervenção coronária percutânea (ICP) primária no tratamento de infarto agudo do miocárdio, quando aplicada no período de tempo ideal,

Tabela 4 - Relação do escore de Killip durante a internação com o procedimento realizado

\begin{tabular}{ccccc}
\hline & $\begin{array}{c}\text { Trombolítico } \\
<\mathbf{6} \text { horas }\end{array}$ & $\begin{array}{c}\text { Angioplastia } \\
>\mathbf{1 2} \text { horas }\end{array}$ & $\mathbf{p}$ & OR (IC 95\%) \\
\hline Killip & $\mathbf{n = 1 3}$ & $\mathbf{n = 2 8}$ & & \\
\hline I & $12(92,3 \%)$ & $14(50 \%)$ & & $12(1.36-105.13)$ \\
\hline II + III + IV & $1(7,7 \%)$ & $14(50 \%)$ & 0,008 & \\
\hline
\end{tabular}

\section{Discussão}

A síndrome coronariana aguda, especialmenteo IAM com supradesnível de ST, por possuir um caráter incapacitante, por sua alta taxa de mortalidade e prevalência elevada nos países em desenvolvimento,representa uma das questões de saúde pública mais relevantes da atualidade (WHELTON et al., 1995)

Os atrasos frequentes na realização da ICP primária já são um problema de caráter mundial. Grandes estudos (ARMSTRONG et al., 2010; 2013) vêm sendo realizados para fornecer dados adicionais no auxílio da decisão terapêutica primária.

Constitui-se então um desafio permanente das grandes metrópoles: a existência obrigatória de diversos níveis de integração do sistema de saúde para que ocorra a correta condução do paciente até ou seja, em menos de 12 horas, realizada por uma equipe experiente, em um serviço de hemodinâmica e cardiologia intervencionista que seja atuante 24 horas por dia e com um retardo porta/balão menor do que 90 minutos (ANTMAN et al., 2004; KUSHNET et al., 2009; MATTOS et al., 2008), está associada à redução da mortalidade, do reinfarto e dos acidentes vasculares encefálicos quando comparada à terapia trombolítica (DESCH et al., 2010). É indicada nessa situação, como recomendação I, o nível de evidência A (MATTOS et al., 2008).

Contudo, sabe-se que no Brasil uma minoria dos casos é executada de tal forma e são muitos os motivos para o atraso e o funcionamento inadequado desse sistema. Causas específicas relacionadas ao período pré-hospitalar (do início dos sintomas e decisão de procurar atendimentoaté a chegada ao hospital) podem ser citadas, como a não valorização, pelo 
paciente, dos sintomas de dor torácica como sendo infarto (talvez por considerar que infarto é sempre um quadro de elevada gravidade e incomum); atribuição dos sintomas a condições crônicas pré-existentes ou a uma doença comum (gripe, dor muscular etc.); ausência de conhecimento dos benefícios que podem ser obtidos com o tratamento rápido; o atendimento extra-hospitalar de urgência, não disponível a todos de forma homogênea (PIEGAS et al., 2009).

Além disso, ainda é frequente o atraso na chegada do paciente até o serviço de hemodinâmica, principalmente pela falta de logística (inviabilidade de transporte, malha rodoviária inadequada, dificuldade de acesso, trânsito caótico) ou por longa distância entre o paciente e o atendimento. Hospitais dedicados e habilitados muitas vezes aderem apenas parcialmente aos protocolos de atendimento a infartados e/ou falham na capacitação do sistema de atendimento, situação que é mais crítica fora do horário comercial. Somado a isso, temos ainda uma pobre distribuição de centros especializados sobre o território nacional.

$\mathrm{Na}$ tentativa de padronizar uma rede de atendimento ao IAM, já existem trabalhos (CALUZA et al., 2012) que demonstraram de forma objetiva e com resultados satisfatórios a importância de seguir um protocolo que incentive a inserção do uso do fibrinolítico em casos em que o atraso em submetero pacienteà ICP primária é evidente.

O enfoque destes primeiros trabalhos está nos grandes centros urbanos, onde o transito caótico e os engarrafamentos extensos são a principal causa do início tardio do tratamento. Em nosso estudo, entretanto, queremos levar à discussão o fato de que em muitas áreas do Brasil há uma pobreza na distribuição de centros especializados e grandes distâncias precisam ser percorridas para que o paciente infartado chegue a um hospital que possua oserviço de hemodinâmica intervencionista.

Para avaliar os diferentes desfechos entre os grupos RQ e RM e tentar demonstrar os benefícios do uso do fibrinolítico quando há atraso na implementação da ICP primária, comparou-se a classificação de Killip-Kimbal do pacientecom o intervalo de tempo até a efetivação da intervenção terapêutica.

A classificação de Killip foi aplicada na avaliação clínica na fase pós-aguda do IAM. Durante o período de evolução do infarto, a migração dos portadores de uma classe mais baixa para uma mais alta está associada independentemente com elevação da mortalidade dentro dos primeiros 30 dias (KILLIP; KIMBALL, 1967).

Os resultados encontrados nos dados da Tabela 4 confirmam que se a perspectiva de o paciente ser submetido a angioplastia for desfavorável, é preferível fazer trombolítico $(\mathrm{p}=0.008)$ com OD de 12 , mostrando que a possibilidade do paciente desenvolver Killip (II, III e IV) é 12 maior na angioplastia após 12 horas quando comparada ao trombolítico em menos 6 horas de evolução do IAM.

Nosso estudo tem a perspectiva futura da criação, por meio da Secretaria de Saúde do Estado, de um fluxograma de atendimento ao IAMCSST para os Campos Gerais, região do Paraná que abrange 13 municípios e possui apenas dois hospitais com serviço de hemodinâmica ativo 24 horas por dia, ambos localizados na cidade de Ponta Grossa.

\section{Conclusão}

A ICP primária é a estratégia preferencial como terapia de reperfusão no IAMCSST sempre que o retardo porta/balão seja inferior a 90 minutos e viável por questões logísticas. Quando é efetivo e imediato, o procedimento leva a melhor sobrevida hospitalar e menor número de complicações.

Entretanto, por meio dos resultados conseguimos estabelecer a importância da utilização da terapia fibrinolítica sempre que o retardo para a realização da angioplastia for elevado, e que esta conduta garante a melhor evolução clínica do paciente.

\section{Agradecimentos}

À professora Dra. Elise Souza dos Santos Reis, pela orientação, colaboração e paciência.

\section{Vinculação Acadêmica}

Este artigo corresponde ao Trabalho de Conclusão de Curso (TCC) de graduação em Medicina de Bruno Ribeiro, da Universidade Estadual de Ponta Grossa. 


\section{Referências}

ANTMAN, E. M. et al. ACC/AHA guidelines for the management of patients with ST-elevation myocardial infarction-executive summary: a report of the American College of Cardiology/ American Heart Association Task Force on Practice Guideline (Writing Committee to Revise the 1999 Guidelines for the Management of Patients With Acute Myocardial Infarction). Circulation, v.100, n.5, p.588-636, 2004.

ARMSTRONG, P. W. et al. A comparison of pharmacologic therapy with/without timely coronary intervention vs. primary percutaneous intervention early after ST-elevation myocardial infarction: the WEST (Which Early STElevation Myocardial Infarction Therapy) study. European Heart Journal, v.27, p.1530-1538, 2006.

The Strategic Reperfusion Early After Myocardial Infarction (STREAM) study. American Heart Journal, v.160, n.1, p.30-35, 2010.

Fibrinolysis or primary PCI in ST-segment elevation myocardial infarction. New England Journalof Medicine, 2013.

BORGIA, F.et al. Early routine percutaneous coronary intervention after fibrinolysis vs. standard therapy in STsegment elevation myocardial infarction: a meta-analysis. European Heart Journal, v.31, p.2156-2169, 2010.

CALUZA, A. C. V. et al. Rede de Infarto com Supradesnivelamento de ST: sistematização em 205 casos diminui eventos clínicos na Rede Pública. Arquivos Brasileiros de Cardiologia, v.99, n.5, p.1040-1048, 2012.

DENKTAS, A. E. et al. Total Ischemic Time: The Correct Focus of Attention for Optimal ST-Segment Elevation Myocardial Infarction Care. Journal of American College Cardiology Interventions, v.4, n.6, p.599-604, 2011.

$\mathrm{DESCH}, \mathrm{S}$. et al. Timing of invasive treatment after fibrinolysis in ST elevation myocardial infarction - a metaanalysis of immediate or early routine versus deferred or ischemia-guided randomised controlled trials. Heart, v.96, p.1695-1702, 2010.

HUYNH, T. et al.Comparison of primary percutaneous coronary intervention and fibrinolytic therapy in STsegment-elevation myocardial infarction: Bayesian hierarchical meta-analyses of randomized controlled trials and observational studies.Circulation, v.119, n.24, p.31013109, 2009.

KILLIP, T.;KIMBALL, J. T. Treatment of myocardial infarction in a coronary care unit.A two year experience with 250 patients. American Journal of Cardiology, v.12, p.457-464, 1967.
KUSHNER, F. G. et al. 2009 Focused Updates: ACC/ AHA Guidelines for the Management of Patients With ST-Elevation Myocardial Infarction (updating the 2004 Guideline and 2007 Focused Update) and ACC/AHA/ SCAI Guidelines on Percutaneous Coronary Intervention (updating the 2005 Guideline and 2007 Focused Update): a report of the American College of Cardiology Foundation/ American Heart Association Task Force on Practice Guidelines. Circulation, v.120, n.22, p.2271-2306, 2009.

LAMBERT, L.et al. Association Between Timeliness of Reperfusion Therapy and Clinical Outcomes in STElevation Myocardial Infarction. Journal of the American Medical Association, v.303, n.21, p.2148-2155, 2010.

MATTOS, L. A. et al. Diretrizes da Sociedade Brasileira de Cardiologia: intervenção coronária percutânea e métodos adjuntos diagnósticos em cardiologia intervencionista. Revista Brasileira de Cardiologia Invasiva, 2 ed., v.16, n.2supl 2, p.9-88, 2008.

MURRAY, C. J. L.;LOPEZ, A. D. The global Burden of disease: a comprehensive assessment of mortality and disability from disease, injuries and risk factors in 1990 and projected to 2020. EUA: Harvard School of Health, 1996.

PIEGAS, L. S. et al. IV Diretriz da Sociedade Brasileira de Cardiologia sobre Tratamento do Infarto agudo do Miocárdio com Supradesnível do Segmento ST. Arquivos Brasileiros de Cardiologia, v.93, n.6 supl. 2, p.179-264, 2009.

VAN DE WERF, F. et al. Management of acute myocardial infarction in patients presenting with persistent ST-segment elevation: the task force on the management of ST-segment elevation acute myocardial infarction of the European Society of Cardiology. European Heart Journal, v.29, p.2909-2945, 2008.

WHELTON, P. K.et al. The challenge of hypertension and atherosclerotic cardiovascular disease in economically developing countries. High Blood Press, v.4, p.36-45, 1995.

WORLD HEALTH ORGANIZATION. Causes of death 2008: data sources and methods. Geneva. World Health Organization, 2010.

WORLD HEALTH ORGANIZATION.Global status report on noncommunicable diseases 2010. Geneva, World Health Organization, 2011. 